\title{
Recent Advances in Systemic Therapies for Advanced Hepatocellular Carcinoma
}

\author{
Gabriella Aitcheson ${ }^{1} \cdot$ Anjana Pillai $^{2} \cdot$ Bassam Dahman $^{3} \cdot$ Binu V John $^{4}$ (B) \\ Accepted: 3 January 2021 / Published online: 1 February 2021 \\ (C) This is a U.S. government work and not under copyright protection in the U.S.; foreign copyright protection may apply 2021
}

\begin{abstract}
Purpose of Review This paper aims to summarize the data of recently completed and key ongoing clinical trials of systemic agents for advanced hepatocellular carcinoma (aHCC). In particular, the review focuses on ongoing checkpoint inhibitor combination trials and promising studies combining tyrosine kinase inhibitors with checkpoint inhibitors.

Recent Findings The recently approved combination of atezolizumab and bevacizumab based on the IMbrave150 trial has shown the most potential with the highest overall survival of any systemic agent in HCC to date, surpassing sorafenib. Despite COVID19 delays, other promising trials that involve combining VEGF-directed therapy and checkpoint inhibition, cancer vaccines, phosphatidylserine, YIV-906, and oncolytic and immunotherapeutic vaccinia virus are actively recruiting patients.

Summary After almost a 10-year dormancy, the list of potential systemic treatment options for aHCC is growing rapidly. Given the promising data from the IMbrave150 trial, the combination of atezolizumab and bevacizumab is now the new first-line therapy. We discuss the change in landscape, the new second- and third-line systemic treatments in aHCC, and the ongoing clinical trials for newer agents including combination therapies.
\end{abstract}

Keywords Atezolizumab $\cdot$ Bevacizumab $\cdot$ Immunotherapy $\cdot$ Clinical trials $\cdot$ Tyrosine kinase inhibitors

\section{Introduction}

Hepatocellular carcinoma has the second-lowest survival rate of major cancers in the USA with its 5-year survival of $18 \%$ trailing behind only pancreatic cancer in mortality [1]. Tumor resection, ablation, and liver transplant are potentially curative options for those with early-stage disease, while locoregional modalities such as trans-arterial chemoembolization and selective internal radiation therapy with yttrium-90 are more utilized in intermediate-stage

This article is part of the Topical Collection on Hepatic Cancer

Binu V John

Binu.John@va.gov

1 Department of Internal Medicine, Jackson Memorial Hospital, Miami, FL, USA

2 Division of Gastroenterology, University of Chicago, Chicago, IL, USA

3 Department of Health Behavior and Policy, Virginia Commonwealth University, Richmond, VA, USA

4 Division of Hepatology, Bruce W Carter VA Medical Center, Miami, FL 33125, USA disease confined to the liver [2, 3]. For those with advanced hepatocellular carcinoma (aHCC), in which there is macrovascular invasion or extrahepatic spread yet preserved liver function, systemic therapy has been the standard of care [4]. While the overall survival of all-comers with HCC has improved in the past few years, this appears to be driven primarily by stage migration, whereas the survival of patients with advanced cancer has remained unchanged [5]. However, with the approval of a series of new agents over the past 3 years, and the most recent approval of atezolizumab and bevacizumab which together have shown the highest overall survival of any systemic agent in HCC to date, there is substantial excitement and anticipation in the field [6]. In this paper, we review data of ongoing and recently completed trials and discuss upcoming agents that show the greatest promise in the field.

\section{The Landscape of FDA-Approved Systemic Therapies and Key Trials}

Systemic therapy became established as the mainstay in aHCC treatment following the US Food and Drug 
Administration (FDA) approval of the multikinase inhibitor sorafenib in 2007 [7]. There has been a significant expansion in the availability of newer tyrosine kinase inhibitors and checkpoint inhibitors over the past 3 years. While lenvatinib and sorafenib are approved first-line agents, the combination of atezolizumab and bevacizumab is the new standard of care for first-line therapy for aHCC. Tyrosine kinase inhibitors (TKIs) including regorafenib, cabozantinib, and ramucirumab, as well as immune checkpoint inhibitors, nivolumab and pembrolizumab, comprise the second-line therapy options (Table 1) $[8,9]$.
Atezolizumab and Bevacizumab-the New First-line Standard of Care

The recently published phase III IMbrave150 study (NCT03434379) used a combination of drugs for targeted synergy combining a programmed cell death ligand 1 (PDL1) inhibitor atezolizumab and VEGFR inhibitor bevacizumab against sorafenib as a first-line agent for HCC [10]. Atezolizumab and bevacizumab are currently also approved to be used in conjunction with chemotherapeutic agents, carboplatin and paclitaxel, for treatment of non-squamous non-small cell lung cancer. Various studies
Table 1 Currently FDAapproved systemic therapies for advanced hepatocellular carcinoma
FDA-approved systemic therapies for advanced hepatocellular carcinoma

\begin{tabular}{|c|c|c|c|c|c|}
\hline Study name & Drug & Control & $\begin{array}{l}\text { Trial } \\
\text { phase }\end{array}$ & Year completed & $\begin{array}{l}\text { Hazard ratio ( } 95 \% \\
\mathrm{CI} \text { ) and overall } \\
\text { survival (months) }\end{array}$ \\
\hline \multicolumn{6}{|l|}{ First-line } \\
\hline SHARP & Sorafenib & Placebo & $\begin{array}{c}\text { Phase } \\
\text { II }\end{array}$ & 2008 & $\begin{array}{c}0.69(0.55-0.87) \\
10.7 \text { vs } 7.9\end{array}$ \\
\hline $\begin{array}{l}\text { Asia-Pacific } \\
\text { study }\end{array}$ & Sorafenib & Placebo & $\begin{array}{c}\text { Phase } \\
\text { III }\end{array}$ & 2009 & $\begin{array}{l}0.68(0.50-0.93) ; \\
\quad 6.5 \text { vs } 4.2\end{array}$ \\
\hline REFLECT & Lenvatinib & Sorafenib & $\begin{array}{c}\text { Phase } \\
\text { III }\end{array}$ & Ongoing (Jul 2020) & $\begin{array}{l}0.92(0.79-1.06) ; \\
\quad 13.6 \text { vs } 12.3\end{array}$ \\
\hline IMbrave 150 & $\begin{array}{l}\text { Atezolizumab } \\
\quad+ \\
\quad \text { bevacizumab }\end{array}$ & Sorafenib & $\begin{array}{c}\text { Phase } \\
\text { III }\end{array}$ & Ongoing (Jun 2022) & $\begin{array}{l}0.58(0.42-0.79) \\
\text { 6-mo OS: } 84.8 \% \\
\text { vs } 67.2 \%\end{array}$ \\
\hline & & & & & $\begin{array}{l}\text { 12-mo OS: } 72.2 \% \\
\text { and } 54.6 \%\end{array}$ \\
\hline \multicolumn{6}{|l|}{ Second-line } \\
\hline RESORCE & Regorafenib & Placebo & $\begin{array}{l}\text { Phase } \\
\text { III }\end{array}$ & 2019 & $\begin{array}{l}0.63(0.50-0.79) \\
10.6 \text { vs } 7.8\end{array}$ \\
\hline CELESTIAL & Cabozantinib & Placebo & $\begin{array}{c}\text { Phase } \\
\text { III }\end{array}$ & 2019 & $\begin{array}{l}0.76(0.63-0.92) \\
10.2 \text { vs } 8.0\end{array}$ \\
\hline REACH & Ramucirumab & Placebo & $\begin{array}{c}\text { Phase } \\
\text { III }\end{array}$ & 2015 & $\begin{array}{l}0.87(0.72-1.05) \\
9.2 \text { vs } 7.6\end{array}$ \\
\hline REACH-2 & Ramucirumab & Placebo & $\begin{array}{c}\text { Phase } \\
\text { III }\end{array}$ & Ongoing (Dec 2021) & $\begin{array}{l}0.71(0.53-0.95) \\
8.5 \text { vs } 7.3\end{array}$ \\
\hline CheckMate 040 & Nivolumab & N/A & $\begin{array}{r}\text { Phase } \\
\text { I/II }\end{array}$ & Ongoing (Apr 2022) & - \\
\hline $\begin{array}{l}\text { CheckMate } \\
040 \text { - cohort } 4\end{array}$ & $\begin{array}{l}\text { Nivolumab + } \\
\text { ipilimumab }\end{array}$ & N/A & $\begin{array}{r}\text { Phase } \\
\text { I/II }\end{array}$ & Ongoing (Apr 2022) & $\begin{array}{l}\text { - } \\
23 \text { in arm A, 12-13 } \\
\quad \text { in B and C }\end{array}$ \\
\hline CheckMate 459 & Nivolumab & Sorafenib & $\begin{array}{c}\text { Phase } \\
\text { III }\end{array}$ & Ongoing (Dec 2021) & $\begin{array}{c}0.85(0.72-1.02) \\
16.4 \text { vs } 14.7\end{array}$ \\
\hline KEYNOTE-224 & Pembrolizumab & N/A & $\begin{array}{c}\text { Phase } \\
\text { II }\end{array}$ & Ongoing (May 2021) & - \\
\hline KEYNOTE-240 & Pembrolizumab & $\begin{array}{l}\text { Placebo + } \\
\text { best } \\
\text { support- } \\
\text { ive care }\end{array}$ & $\begin{array}{c}\text { Phase } \\
\text { III }\end{array}$ & Ongoing (Jun 2021) & $\begin{array}{l}0.78(0.61-1.00) \\
13.9 \text { vs } 10.6\end{array}$ \\
\hline
\end{tabular}


are underway exploring the effects of the two monoclonal antibodies in other solid tumors though none seems to have achieved the remarkable level of effects seen in the IMbrave150 trial. As a PD-L1 inhibitor, atezolizumab prevents the immune checkpoint action in which $\mathrm{T}$ cell response against cancer proliferation is deactivated, while bevacizumab prevents the angiogenesis resulting from VEGFR activation and blocks additional blood supply to the tumor. In the IMbrave150 study, systemic treatmentnaïve patients with locally advanced or metastatic and/or unresectable HCC were divided into two arms, one receiving atezolizumab $1200 \mathrm{mg}$ IV and bevacizumab $15 \mathrm{mg} / \mathrm{kg}$ IV every 3 weeks while the other received standard-of-care sorafenib $400 \mathrm{mg}$ twice daily. Patients were treated until they reached unacceptable toxicity or experienced loss of clinical benefit. The primary endpoints of focus were overall survival and independent review facility-assessed progression-free survival with objective response rate as a secondary outcome $[11 \bullet \cdot]$.

Of the 501 patients included in the study, 336 received the dual combination while 165 received sorafenib. The 6and 12 -month overall survival rates were $84.8 \%$ and $67.2 \%$ with atezolizumab-bevacizumab and $72.2 \%$ and $54.6 \%$ with sorafenib (HR $0.58 ; 95 \%$ CI $0.42-0.79 ; p=0.0006$ ). The median progression-free survival with atezolizumabbevacizumab was 6.8 months versus 4.3 months with sorafenib (HR $0.59 ; 95 \%$ CI $0.47-0.76 ; p<0.0001$ ). Atezolizumab-bevacizumab had more frequent serious adverse events. The most common grade 3 or 4 adverse event was hypertension in $15.2 \%$. As one would anticipate with bevacizumab, upper GI bleeding was a common adverse event observed in $7 \%$ and led to drug discontinuation in $0.03 \%$. All patients in the trial had been selected after initial screening endoscopy and those with varices were treated before enrollment. This is significant because $40 \%$ of the study population was from Asia (excluding Japan) and the most common etiology of liver disease was hepatitis B in $49 \%$, a group where HCC commonly occurs in the absence of cirrhosis. This is corroborated by the fact that only $26 \%$ of the subjects had varices at baseline. One can infer in a Western population where there is a higher incidence of portal hypertension and cirrhosis that the instance of GI bleed is likely to be higher. Atezolizumab-bevacizumab also delayed the time to deterioration of patient-reported quality of life to 11.2 months versus 3.6 months with sorafenib. Delays in time to deterioration of patient-reported physical functioning and role functioning in the dual-drug regimen versus sorafenib were 13.1 months versus 4.9 months and 9.1 months versus 3.6 months, respectively $[11 \bullet \cdot 12]$. This combination of atezolizumab and bevacizumab marks territory as the first treatment found to be superior to sorafenib and establishes itself as a new standard of care for HCC.

\section{First-line Alternative Agents-Multikinase Inhibitors}

The SHARP trial (NCT00105443) helped open the door for systemic therapies in the treatment of aHCC. In this phase III randomized controlled trial, aHCC patients with Child-Pugh class A saw an increased median survival to 10.7 months on sorafenib versus 7.9 months on placebo [13]. The subsequent smaller-scale Asia-Pacific trial (NCT00492752) reinforced these positive outcomes with median overall survivals of 6.5 months on sorafenib compared to 4.2 months on placebo [14].

In the decade following, multiple small molecule inhibitors and treatment approaches were developed to treat aHCC but no therapy matched nor surpassed sorafenib in survival outcomes and safety. It was not until the REFLECT trial (NCT01761266) exhibited the non-inferiority of lenvatinib, another multikinase inhibitor, to sorafenib that first-line treatment options again saw possible expansion. Lenvatinib conferred a 13.6-month median survival versus 12.3 months with sorafenib in patients with unresectable hepatocellular carcinoma and Child-Pugh class A [15]. A recent post hoc analysis of baseline liver function-measured by albumin-bilirubin (ALBI) grade or Child-Pugh (CP) score - and efficacy and safety outcomes showed overall greater benefit from lenvatinib over sorafenib regardless of ALBI grade or ChildPugh score [16]. The median overall survival in CP-5 patients was 15.3 months with lenvatinib versus 14.2 months with sorafenib. Similarly, ALBI-1 patients had a survival of 17.4 months versus 14.6 months with sorafenib. Both saw notable decreases in survival with CP-6 and ALBI-2 with lenvatinib conferring survival of 9.4 months versus 7.9 months and 8.6 versus 7.7 months, respectively. Across all lenvatinib subgroups except ALBI-2, progression-free survival was around 7.4 months versus approximately 3.6 months with sorafenib. Lenvatinib is associated with a lower incidence of hand-foot syndrome but a higher incidence of hypertension, compared to sorafenib, but there is little in terms of efficacy, to prefer any one agent over the other.

\section{Second-line Agents}

\section{Multikinase Inhibitors}

The RESORCE trial (NCT01774344) showed the efficacy of the multikinase inhibitor regorafenib both as a second-line agent and as a treatment option after sorafenib therapy failure [17]. Regorafenib gave a median survival of 10.6 months compared to 7.8 months on placebo. Further analysis showed the median survival from sorafenib initiation to death was 26.0 months with regorafenib in succession versus 19.2 months with placebo [18]. In the CELESTIAL trial 
(NCT01908426), another oral multikinase inhibitor, cabozantinib, was studied in patients who had undergone systemic therapy with one or two prior regimens [19]. The median survival on cabozantinib was 10.2 months versus 8.0 months on placebo, showing that cabozantinib could function as an effective second- or third-line agent.

While the initial REACH trial (NCT01140347) comparing the anti-VEGFR-2 monoclonal antibody ramucirumab to placebo in patients who had already received sorafenib did not display significant survival benefit (9.2 months vs 7.6 months; HR 0.87 (95\% CI $0.72-1.05)$ ), the REACH-2 trial (NCT02435433) helped to carve a role in a subset of aHCC patients [20]. A REACH trial sub-analysis showed patients with an AFP level $\geq 400 \mathrm{ng} / \mathrm{mL}$ who received ramucirumab had a median survival of 7.8 months compared to 4.2 months on placebo (HR $0.67 ; 95 \%$ CI $0.51-0.90$ ), while those with an AFP $<400 \mathrm{ng} / \mathrm{mL}$ had a 10.1-month median survival versus 11.8 months with placebo (HR 1.09; 95\% CI 0.84-1.43). REACH-2 followed a similar study design to its predecessor but only included those with AFP concentrations $\geq 400 \mathrm{ng} /$ $\mathrm{mL}$. Data was pooled with REACH participants with AFP levels $\geq 400 \mathrm{ng} / \mathrm{mL}$ and yielded a median overall survival of 8.5 months with ramucirumab and 7.3 months with placebo, ultimately proving another second-line agent [21].

\section{Checkpoint Inhibitors}

Immunotherapy has further expanded treatment options for advanced hepatocellular carcinoma. Based on the CheckMate 040 (NCT01658878) and KEYNOTE-224 (NCT02702414) trials, the FDA granted accelerated approval of programmed cell death 1 (PD-1) inhibitors nivolumab and pembrolizumab, respectively $[22,23]$. In the phase I/II CheckMate 040 trial studying nivolumab, the doseexpansion phase had a $20 \%$ objective response rate with three complete responses and 39 partial responses and ultimately a $64 \%$ disease control rate [24]. The dose-escalation phase had an objective response rate of $15 \%$ and a 15.0 -month median overall survival. Additionally, nivolumab was found to have a manageable safety profile and acceptable tolerability.

The phase III randomized multicenter CheckMate 459 trial (NCT02576509) intended to compare nivolumab to sorafenib as an evaluation for potential as a first-line agent, but, per a Bristol Myers Squibb press release in June 2019, the trial ultimately failed to achieve statistical significance for overall survival based on their pre-specified analysis [25]. Data presented at the 2020 ASCO Gastrointestinal Cancers Symposium (ASCO GI 2020) showed the median overall survival at 16.4 months for those on nivolumab and 14.7 months on sorafenib with a hazard ratio of $0.85(p=0.0752)$ [26]. The overall response rate was $15 \%$ for nivolumab and $7 \%$ for sorafenib. The FACT-Hep showed favor of nivolumab over sorafenib in health-related quality of life with regard to FACT-
Hep total, physical well-being, and functional wellbeing scores. Time to deterioration was found to be significantly delayed with nivolumab for the abovementioned measures. Although statistical significance was not achieved for overall survival, per the analysis, nivolumab leads to clinical improvement and a more positive impact on the quality of life.

In the phase II KEYNOTE-224 trial (NCT02702414), aHCC patients who had already undergone sorafenib therapy demonstrated a $17 \%$ objective response and 12.9 -month median survival with pembrolizumab [27]. A 2-year follow-up analysis presented at ASCO GI 2020 showed an objective response rate of $18.3 \%$ up from $17 \%$ in the original study results [28]. The median duration of response was 21 months with $77 \%$ of responses lasting 12 months or longer, up from $61.4 \%$ in the original study. The median progression-free survival was 4.9 months with $11.3 \%$ attaining progression-free survival at 24 months and median overall survival was 13.2 months with $30.8 \%$ reaching overall survival of 24 months. Treatment-related adverse events (TRAEs) occurred in $73.1 \%$ of patients with the most common symptoms being fatigue, increased aspartate aminotransferase, pruritus, diarrhea, and grade 3 or higher adverse events occurring in $26.0 \%$ of patients.

A subsequent randomized double-blind phase III study of pembrolizumab, KEYNOTE-240 (NCT02702401), compared pembrolizumab with best supportive care in previously treated aHCC patients [29]. In the overall cohort, pembrolizumab improved the median overall survival to 13.9 months versus 10.6 months on placebo. Disease progression occurred in $62.2 \%$ of patients on pembrolizumab versus $74.1 \%$ in the placebo group. The objective response rate in the overall cohort was $18.3 \%$ with pembrolizumab and $4.4 \%$ with placebo, while analysis of the Asian subgroup showed 20.6\% versus $2.0 \%$ [30]. Although the overall survival and progression-free survival improved with pembrolizumab, they did not meet the study's predefined values of significance.

\section{Old Drugs and New Combinations}

While further studies are being done on the efficacy and safety of first- and second-line agents currently available for treatment, the focus of other studies has shifted to various combinations of these previously vetted VEGF-directed therapies and checkpoint inhibitors.

\section{Combining Immunotherapy-Anti-PD-1 and Anti- CTLA-4}

The combination of nivolumab's PD-1 inhibition activity with ipilimumab, a cytotoxic T lymphocyte antigen 4 (CTLA-4) inhibitor, was initially utilized in patients with advanced melanoma. Targeting these two immune checkpoints has been 
shown to stimulate the further enhancement of the immune system's response against cancer beyond the effects of a singular agent, and thus, use has expanded to other solid tumors. Most recently, this combination of checkpoint inhibitors received accelerated approval from the FDA for patients with hepatocellular carcinoma who failed treatment with sorafenib based on data from cohort 4 of the CheckMate 040 trial [31]. Originally presented at the 2019 American Society of Clinical Oncology congress (ASCO 2019), this study reported primary efficacy and safety of nivolumab-ipilimumab combination therapy with three dosing variations in sorafenibexperienced patients [32]. All three groups demonstrated an objective response rate of $31-32 \%$ with cumulatively seven patients showing complete response and a 17-month median duration of response. The disease control rate ranged from 43 to $54 \%$ and the 24-month overall survival rate ranged 30 $48 \%$. The combination was generally well tolerated with $37 \%$ of patients experiencing grade 3-4 TRAEs, the most common of which were pruritus and rash. A follow-up subgroup analysis was later presented at ASCO GI 2020, which re-demonstrated the objective response rate of $31 \%$, the median duration of response of 17 months, and the disease control rate of $49 \%$ [33]. The 30-month overall survival rate was consistent with previously reported results at $37 \%$. Tolerability also remained consistent at a rate of $37 \%$ grade 3-4 TRAEs, but the most common became aspartate aminotransferase, and lipase increases. As this combination now enters the market, additional clinical information will be gleaned.

\section{Combining VEGF-Directed Therapy and Checkpoint Inhibition}

Another combination of FDA-approved medications is looking to create a synergistic effect between checkpoint inhibitors' enhancement of anticancer immune response and VEGF inhibition's antitumor angiogenesis effects. The CABO cohort of the CheckMate 040 trial compares a nivolumab-cabozantinib-ipilimumab combination triple therapy versus dual therapy with nivolumab-cabozantinib alone in both sorafenib-naïve and sorafenib-experienced patients [34]. The median progression-free survival was 5.5 months for the two-drug regimen and 6.8 months for the three-drug regimen. The median overall survival was not reached in either arm at the time of analysis. The disease control rate was $81 \%$ in the two-drug arm and $83 \%$ in the three-drug arm. Grade 3-4 TRAEs were reported in 15 of the 36 patients in the nivolumab-cabozantinib arm leading to discontinuation in one, while TRAEs occurred in 25 of the 35 patients in the nivolumab-cabozantinib-ipilimumab arm leading to discontinuation in 7 patients. Ultimately, both therapy combinations led to clinically meaningful responses and, despite the increased rate of TRAEs in the three-drug arm, most adverse events were ultimately manageable, making both viable options for treatment.

\section{Overview of Trials in Progress}

A slew of trials in progress presented at the ASCO 2020 Gastrointestinal Cancers Symposium have been exploring novel combinations of drugs as well as new compounds with novel targets (Table 2).

\section{Other Ongoing Phase I/II Studies Combining Anti- VEGF and Immune Checkpoint Inhibitors}

Study 117 (NCT03418922), a phase Ib open-label Japanese study, is combining VEGF-directed therapy and checkpoint inhibition to explore the tolerability and safety of a combined lenvatinib-nivolumab regimen [35•]. Early data shows no drug-limiting toxicities, but all 30 participants experienced treatment-emergent adverse events (TEAEs) with $56.7 \%$ having palmar-plantar erythrodysesthesia and $53.5 \%$ having dysphonia. Overall, adverse events were manageable and $76.7 \%$ had an overall response rate, $63.3 \%$ had a partial response, $20 \%$ had stable disease, and $13.3 \%$ had a complete response. The final data collection for primary outcome measures is ongoing.

Another VEGF-immune checkpoint inhibitor combination study is a multicenter, phase Ib/II study (NCT03211416) looking at the synergistic effects of sorafenib and pembrolizumab [36•]. The 27 study patients will take sorafenib with pembrolizumab every 3 weeks after tolerating sorafenib for 1 month. As of September 2019, 13 patients are enrolled with nine already receiving the combination treatment. The endpoints of interest will be the overall response rate, safety, overall survival, and progression-free survival.

A single-center phase II randomized control trial at NYU (NCT04050462) is looking at combining nivolumab with either anti-IL-8 (BMS-986253) or cabiralizumab to potentiate the positive effects seen by the checkpoint inhibitor in prior studies [37]. The study expands on the premise demonstrated by some preclinical and translational studies which have shown that IL-8 and tumor-associated macrophages can contribute to the progression of hepatocellular carcinoma as well as the recurrence after treatment. The intended study population is those with Childs A liver function and without prior systemic treatment.

\section{Phase I/II Trials with Novel Targets}

A currently ongoing single-center, phase I Japanese trial, CRESCENT 1 (jRCT2031190072), is taking on an avantgarde approach with their novel cancer vaccine CYT001 [38]. Researchers used an artificial intelligence-based 
Table 2 Ongoing clinical trials of systemic therapies

\begin{tabular}{|c|c|c|c|c|c|}
\hline \multicolumn{6}{|c|}{ Ongoing clinical trials of systemic therapies for advanced hepatocellular carcinoma } \\
\hline Study name & Drug & Control & $\begin{array}{l}\text { Trial } \\
\text { phase }\end{array}$ & $\begin{array}{l}\text { Estimated } \\
\text { primary and } \\
\text { study completion } \\
\text { dates }\end{array}$ & Trial identifier \\
\hline \multicolumn{6}{|l|}{ Phase I } \\
\hline CRESCENT & $\begin{array}{l}\text { CYT001 + } \\
\text { Poly-ICLC + } \\
\text { LAG-3Ig }\end{array}$ & N/A & $\begin{array}{l}\text { Phase } \\
\text { I }\end{array}$ & Unknown & jRCT2031190072 \\
\hline Study 117 & $\begin{array}{c}\text { Lenvatinib + } \\
\text { nivolumab }\end{array}$ & N/A & $\begin{array}{l}\text { Phase } \\
\text { Ib }\end{array}$ & $\begin{array}{l}\text { June } 2021 \\
\text { June } 2021\end{array}$ & NCT03418922 \\
\hline KEYNOTE-524 & $\begin{array}{l}\text { Lenvatinib + } \\
\text { pembrolizumab }\end{array}$ & $\mathrm{N} / \mathrm{A}$ & $\begin{array}{c}\text { Phase } \\
\text { Ib }\end{array}$ & $\begin{array}{l}\text { October } 2019 \\
\text { August } 2021\end{array}$ & NCT03006926 \\
\hline \multicolumn{6}{|l|}{ Phase II } \\
\hline [Unnamed] & $\begin{array}{l}\text { Sorafenib + } \\
\text { pembrolizumab }\end{array}$ & Sorafenib & $\begin{array}{c}\text { Phase } \\
\text { Ib/- } \\
\text { II }\end{array}$ & $\begin{array}{l}\text { September } 2021 \\
\text { September } 2021\end{array}$ & NCT03211416 \\
\hline $\begin{array}{l}\text { CheckMate } \\
040 \text { - CABO } \\
\text { cohort }\end{array}$ & $\begin{array}{l}\text { Nivolumab }+ \\
\text { cabozantinib + } \\
\text { ipilimumab }\end{array}$ & $\begin{array}{l}\text { Nivolumab } \\
\quad+ \\
\text { cabozant- } \\
\text { inib }\end{array}$ & $\begin{array}{r}\text { Phase } \\
\text { I/II }\end{array}$ & $\begin{array}{l}\text { August } 2021 \\
\text { April } 2022\end{array}$ & NCT01658878 \\
\hline [Unnamed] & $\begin{array}{l}\text { Nivolumab }+ \\
\text { BMS-986253 or } \\
\text { cabiralizumab }\end{array}$ & Nivolumab & $\begin{array}{c}\text { Phase } \\
\text { II }\end{array}$ & $\begin{array}{l}\text { August } 2022 \\
\text { August } 2024\end{array}$ & NCT04050462 \\
\hline [Unnamed] & $\begin{array}{l}\text { Sorafenib + } \\
\text { YIV-906 }\end{array}$ & $\begin{array}{l}\text { Sorafenib + } \\
\quad \text { Placebo }\end{array}$ & $\begin{array}{c}\text { Phase } \\
\text { II }\end{array}$ & $\begin{array}{l}\text { December } 2021 \\
\text { February } 2022\end{array}$ & NCT04000737 \\
\hline [Unnamed] & $\begin{array}{c}\text { Pembrolizumab }+ \\
\text { bavituximab }\end{array}$ & $\begin{array}{l}\text { Historical } \\
\text { controls }\end{array}$ & $\begin{array}{c}\text { Phase } \\
\text { II }\end{array}$ & $\begin{array}{l}\text { April } 2021 \\
\text { April } 2022\end{array}$ & NCT03519997 \\
\hline \multicolumn{6}{|l|}{ Phase III } \\
\hline HIMALAYA & $\begin{array}{l}\text { Durvalumab }+/- \\
\text { tremelimumab }\end{array}$ & Sorafenib & $\begin{array}{c}\text { Phase } \\
\text { III }\end{array}$ & $\begin{array}{l}\text { December } 2021 \\
\text { April } 2022\end{array}$ & NCT03298451 \\
\hline COSMIC-312 & $\begin{array}{r}\text { Cabozantinib + } \\
\text { atezolizumab }\end{array}$ & Sorafenib & $\begin{array}{c}\text { Phase } \\
\text { III }\end{array}$ & $\begin{array}{l}\text { June } 2021 \\
\text { December } 2021\end{array}$ & NCT03755791 \\
\hline LEAP-002 & $\begin{array}{l}\text { Lenvatinib }+ \\
\text { pembrolizumab }\end{array}$ & Lenvatinib & $\begin{array}{c}\text { Phase } \\
\text { III }\end{array}$ & $\begin{array}{l}\text { May } 2022 \\
\text { May } 2022\end{array}$ & NCT03713593 \\
\hline PHOCUS & $\begin{array}{l}\text { Sorafenib + } \\
\text { pexastimogene } \\
\text { devacirepvac } \\
\text { (Pexa-Vec) }\end{array}$ & Sorafenib & $\begin{array}{c}\text { Phase } \\
\text { III }\end{array}$ & $\begin{array}{l}\text { December } 2020 \\
\text { December } 2020\end{array}$ & NCT02562755 \\
\hline $\begin{array}{l}\text { RATIONALE } \\
301\end{array}$ & $\begin{array}{l}\text { BGB-A317 } \\
\quad \text { (tislelizumab) }\end{array}$ & Sorafenib & $\begin{array}{c}\text { Phase } \\
\text { III }\end{array}$ & $\begin{array}{l}\text { June } 2021 \\
\text { May } 2022\end{array}$ & NCT03412773 \\
\hline IMbrave150 & $\begin{array}{r}\text { Atezolizumab }+ \\
\text { bevacizumab }\end{array}$ & Sorafenib & $\begin{array}{c}\text { Phase } \\
\text { III }\end{array}$ & $\begin{array}{l}\text { February } 2021 \\
\text { June } 2022\end{array}$ & NCT03434379 \\
\hline
\end{tabular}

prediction system to screen according to proteome, mRNA, and histopathology data from human samples to find sharedantigen peptides to "boost the cancer immunity cycle." This process yielded heat shock protein 70 and glypican 3 as these multi-HLA reactive peptides showed cross-reactivity to HLAA 24:02, 02:01, and 02:06. The study also looked at PolyICLC (Oncovir Inc.) which binds to Toll-like receptor 3 (TLR3) and melanoma differentiation antigen 5 (MDA5) on antigen-presenting cells (APCs) and consequently activates the APCs as well as LAG-3Ig (Immutep Inc.) which binds to the major histocompatibility complex (MHC) class II molecules of APCs and activates them. Together, these two compounds synergistically activate antigen-specific CTL reactions and act as combination adjuvants. The study will assess doselimiting toxicity and safety and response rate via serum evaluation and biomarkers (PD-1, LAG3, TIM3, CTLA-4, etc.) via liver tumor biopsy samples [39].

Phosphatidylserine, another novel target, is an immunosuppressive lipid localized to the inner leaflet of the standard cell membrane, which is externalized to the outer leaflet of the 
plasma membrane in cells lining tumor blood vessels, tumor cells, and exosomes in the tumor microenvironment [40]. One nonrandomized, open-label, multi-site phase II therapeutic trial (NCT03519997) is exploring the utility of combining bavituxumab, a phosphatidylserine-targeting antibody, with pembrolizumab in those with aHCC who have no received prior systemic therapy [41]. The overall response rate, safety, tolerability, overall survival, 6-month progression-free survival, and duration of response will be evaluated.

One currently recruiting, international, multicenter, doubleblind randomized control phase II trial (NCT04000737) looking specifically at hepatitis B-positive patients with aHCC will explore the role of YIV-906. YIV-906 (PHY906, KD018) is a compound derived from the traditional Chinese herbal medicine Huang Qin Tang [42]. The study will look at the combination of sorafenib with YIV-906 given preclinical research showing potential synergistic anticancer activity and reduction of GI toxicity in cancer regimens [43].

\section{Phase III Trials}

Another multicenter randomized phase III study, PHOCUS (NCT02562755), is exploring the utility of pexastimogene devacirepvec (Pexa-Vec, JX-594), an oncolytic and immunotherapeutic vaccinia virus, combined with sorafenib. A prior phase II randomized study in patients with unresectable hepatocellular carcinoma showed that intratumor injections with the high-dose Pexa-Vec treatment were associated with improved overall survival in comparison to its low-dose control with a median of 14.1 months versus 6.7 months [44]. In the PHOCUS trial, patients will receive three biweekly intratumoral injections over the course of 4 weeks, which will then be followed by the administration of sorafenib on week 6 . These patients will be compared to a cohort receiving sorafenib alone. The overall survival, time to progression, progression-free survival, overall response rate, and disease control rate will be evaluated.

The ongoing RATIONALE 301 study (NCT03412773) is comparing PD-1 inhibitor BGB-A317 (tislelizumab) against sorafenib as a potential new immune checkpoint inhibitor to be used in the treatment of advanced HCC. Tislelizumab has a unique edge compared to other PD-1 inhibitors in that it was engineered to avoid the antibody-dependent phagocytosis of macrophages [45]. Prior phase Ia/Ib data (NCT02407990) showed an objective response rate and disease control rate of $12.2 \%$ and $51.0 \%$ in the hepatocellular carcinoma cohort [46]. Of the 49 patients evaluated, six had a partial response, and 19 had stable disease. This multicenter phase III randomized control trial will focus on comparing the overall survival between the two treatments as well as a blinded independent review of objective response rate, progression-free survival, duration of response, and time to progression.
The HIMALAYA trial (NCT03298451) is seeking to break ground as the first trial to test dual immune checkpoint blockade as a first-line treatment for aHCC. It will look at the safety and efficacy of the PD-L1 inhibitor durvalumab with the CTLA-4 inhibitor tremelimumab in patients with unresectable hepatocellular carcinoma who have not received prior systemic therapy [47]. Durvalumab is already approved for unresectable, stage III NSCLC, while tremelimumab is currently being studied in combination with durvalumab for the treatment of various malignancies. Patients will be randomized into four arms evaluating durvalumab monotherapy, two regimens of durvalumab-tremelimumab combination therapy, and sorafenib monotherapy. The overall survival is the primary endpoint with objective response rate, duration of response, disease control rate, progression-free survival, and time to progression as secondary endpoints. Per a press release from the drug manufacturer, AstraZeneca, the FDA has granted this combo an orphan drug designation for HCC treatment [48].

The COSMIC-312 trial (NCT03755791), another first-line agent evaluation, studies aHCC patients who have not received prior systemic treatment to undergo treatment with cabozantinib and atezolizumab [49•]. The study consists of three arms with oral cabozantinib daily and IV atezolizumab every 3 weeks versus sorafenib in the control arm and oral daily cabozantinib as a single-agent exploratory arm. The primary endpoints are overall survival and progression-free survival with objective response rate as a secondary endpoint. As cabozantinib is a tyrosine kinase VEGFR inhibitor already established in HCC treatment and atezolizumab has shown promising results in combination with the VEGFR inhibitor bevacizumab in the IMbrave 150 trial, theoretically this study may yield similar treatment benefits. This study has completed enrollment for new subjects and results are awaited.

The LEAP-002 trial (NCT03713593) also seeks to provide a possible first-line agent by enhancing the efficacy of lenvatinib by adding pembrolizumab [50 ]. The phase $\mathrm{Ib}$ KEYNOTE-524 (NCT03006926) showed that combining lenvatinib and pembrolizumab was generally well-tolerated, demonstrated an objective response rate of $44.8 \%$, and achieved stable disease in $37.3 \%$ [51]. The LEAP-002 trial is a phase III study that builds upon the KEYNOTE-524 trial. One arm will be randomized to receive a lenvatinib dosing according to bodyweight with IV pembrolizumab IV every 3 weeks, while the other will receive lenvatinib dosing according to bodyweight with placebo IV every 3 weeks. Primary outcomes will be progression-free survival and overall survival with objective response rate, duration of response, disease control rate, and time to progression as secondary endpoints. Based on the results from the KEYNOTE-524 trial, the FDA granted breakthrough therapy designation to the lenvatinib-pembrolizumab combination. Further studies will hopefully elucidate its potential as another first-line therapy for aHCC. 


\section{Advanced Hepatocellular Carcinoma}

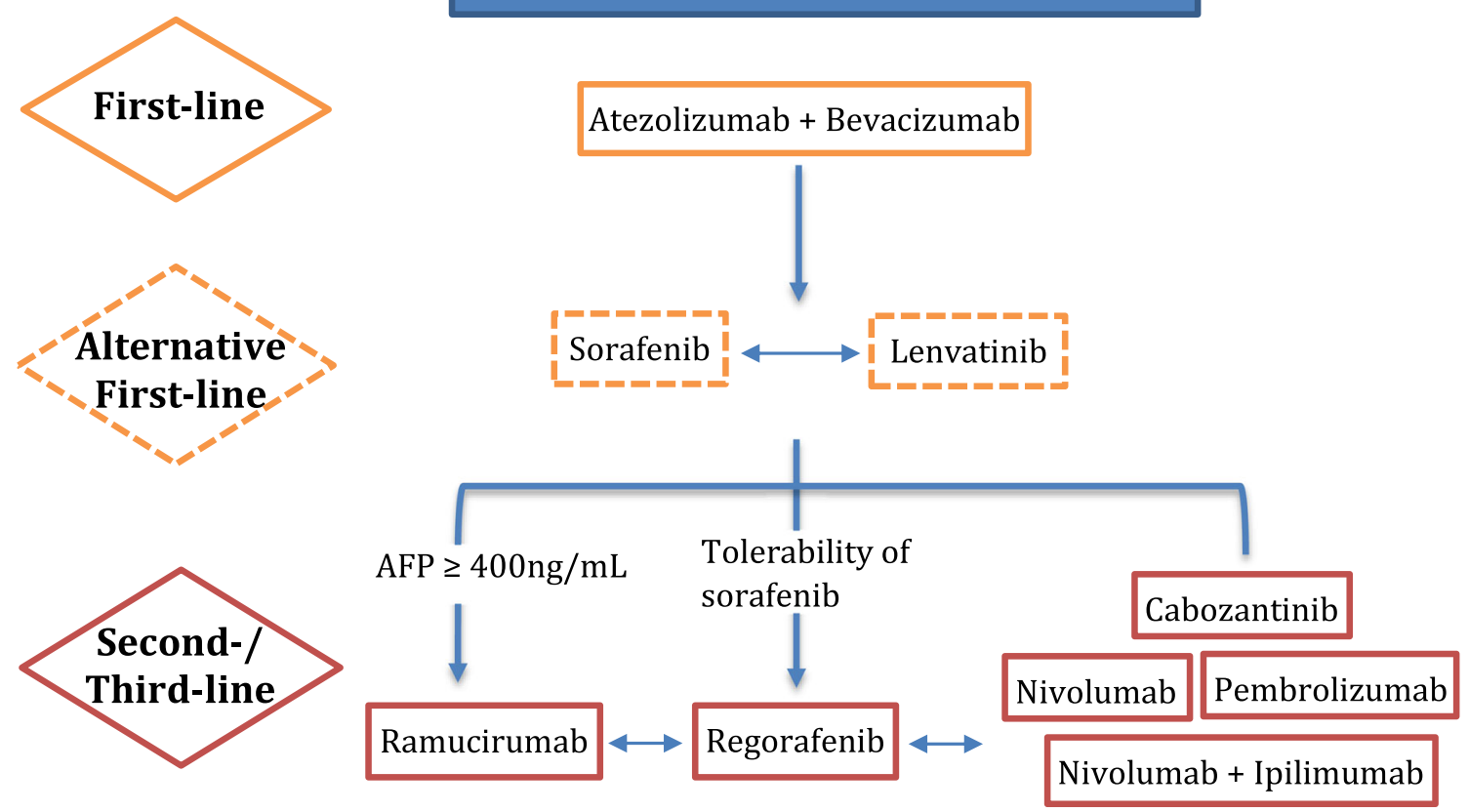

Fig. 1 New progression of treatment lines

\section{Overview of Future Directions and Molecular Targets}

Multiple phase I studies are attempting to change perspective and expand beyond the current repertoire of inhibiting kinases, PD-1, and PDL-1. One phase I trial is investigating the role of a humanized agonist $\operatorname{IgG} 2$ monoclonal antibody to the tumor necrosis factor superfamily receptor OX40, PF04518600 (PF-8600) in aHCC patients (NCT02315066) [52]. Patients received either a low-dose regimen (arm 1) or highdose regimen (arm 2). Of those in arm 1, 50\% achieved stable disease with a mean duration of response at 18.4 weeks, while of those in arm 2, 53\% achieved stable disease with a mean duration of response at 17.4 weeks. In arm 1, 69\% of the participants experienced TRAEs compared to $58 \%$ in arm 2, with rash and pruritus being the most common. For the pharmacodynamic analysis, immunohistochemistry and RNA sequencing showed more pronounced upregulation of OX40 tumor expression and positive changes in gene signatures in arm 1 more so than in arm 2 suggesting a more active antitumor immunostimulatory effect. Nonetheless, both arms were well tolerated and conferred disease control.

Another phase I trial (NCT02716012) is looking at MTLCEBPA, a small activating RNA oligonucleotide that targets the transcription factor $\mathrm{C} / \mathrm{EBPa}$ which regulates myeloid cell differentiation. Early data showed that three out of five patients treated with sorafenib off-study after discontinuing MTL-CEBPA maintained complete radiological response for a period of seven to 18 months and two experienced resolution of their lung metastasis [53]. In subsequent data, 26 patients with underlying hepatitis $\mathrm{B}$ or $\mathrm{C}$ etiology received either combination therapy of MTL-CEBPA with sorafenib or sequential therapy with MTL-CEBPA followed by sorafenib [54]. One patient in the combination group has maintained complete radiological response at 7 months and two in the sequential group have shown stable disease for 3 to 4 months.

In vitro and murine in vivo studies presented at the ASCO GI 2020 highlighted the potential roles of apurinic/ apyrimidinic endonuclease-1, Wnt5a, and mTOR in both prognostications as well as targets for future treatment development [55-57]. Multiple clinical trials currently listed with the US National Library of Medicine database are exploring the possibilities of oncogenic vaccines, immune checkpoint inhibition expanding to cyclin-dependent kinases, and chimeric antigen receptor-modified $\mathrm{T}$ cells. The current treatment landscape appears to be progressing toward rapid expansion if these new targets prove to be fruitful.

\section{Conclusion}

As the list of potential systemic treatment options for advanced hepatocellular carcinoma grows, the upcoming questions that arise will likely involve restructuring the progression among the first-, second-, and third-line treatments as well as the integration of precision medicine. If further data from the IMbrave150 trial continues to remain so profoundly positive, the atezolizumab-bevacizumab combination will likely usurp sorafenib as the first-line treatment. This may mean pushing both sorafenib and lenvatinib into the second line with the 
subsequent treatments taking a corresponding shift back or a re-evaluation on the most efficacious successions of treatments (Fig. 1). Furthermore, with the conversation of oncologic treatment moving toward precision oncology and trials in the works to investigate other prospective targets, therapeutic options that emerge and analysis of preexisting options may see a similar trend.

Funding Services in support of the research project were generated by the VCU Massey Cancer Center Biostatistics Shared Resource, supported, in part, with funding from NIH-NCI Cancer Center Support Grant P30 CA016059.

\section{Compliance with Ethical Standards}

Conflict of Interest Dr. Gabriella Aitcheson declares no conflict of interest.

Dr. Anjana Pillai declares the following conflicts of interest: Medical Advisory Board for Exelixis, Genentetch, Eisai Inc and Speaker's Bureau for Simply Speaking Hepatitis CME series.

Dr. Bassam Dachman declares no conflict of interest.

Dr. Binu John declares the following conflicts of interest: grants from BMS, H3B biosciences, Exelixis, Bayer, Exact Sciences, Glycotest therapeutics, Inc.

Human and Animal Rights and Informed Consent This article does not contain any studies with human or animal subjects performed by any of the authors.

Disclaimer The authors prepared this work in their personal capacity. The opinions expressed in this article are the author's own and do not reflect the view of Department of Veterans Affairs, or the United States government.

\section{References}

Papers of particular interest, published recently, have been highlighted as:

- Of importance

•- Of major importance

1. Siegel RL, Miller KD, Jemal A. Cancer statistics, 2020. CA A Cancer J Clin. 2020;70:7-30. https://doi.org/10.3322/caac.21590.

2. Li D, Sedano S, Allen R, Gong J, Cho M, Sharma S. Current treatment landscape for advanced hepatocellular carcinoma: patient outcomes and the impact on quality of life. Cancers (Basel). 2019;11(6):841. https://doi.org/10.3390/cancers11060841.

3. Villanueva A. Hepatocellular carcinoma. N Engl J Med. 2019;380: 1450-62. https://doi.org/10.1056/NEJMra1713263.

4. Likhitsup A, Razumilava N, Parikh ND. Treatment for advanced hepatocellular carcinoma: current standard and the future. Clinical Liver Dis. 2019;13:13-9. https://doi.org/10.1002/cld.782.

5. De Toni EN, Schlesinger-Raab A, Fuchs M, et al. Age independent survival benefit for patients with hepatocellular carcinoma (HCC) without metastases at diagnosis: a population-based study. Gut. 2020;69:168-76. https://doi.org/10.1136/gutjnl-2018-318193.

6. Kelley RK. Atezolizumab plus bevacizumab - a landmark in liver cancer. N Engl J Med. 2020;382:1953-5. https://doi.org/10.1056/ NEJMe2004851.
7. Lang L. FDA approves sorafenib for patients with inoperable liver cancer. Gastroenterology. 2008;134(2):379-80. https://doi.org/10. 1053/j.gastro.2007.12.037.

8. Grieb BC, Goff LW, Goyal L, Denlinger CS. Evolving landscape of systemic therapy for hepatocellular carcinoma: breakthroughs, toxicities, and future frontiers. Am Soc Clin Oncol Educ Book. 2019;39:248-60. https://doi.org/10.1200/EDBK 237555.

9. Couri T, Pillai A. Goals and targets for personalized therapy for HCC. Hepatol Int. 2019;13:125-37. https://doi.org/10.1007/ s12072-018-9919-1.

10. Cheng A-L, Qin S, Ikeda M, Galle P, Ducreux M, Zhu A, et al. IMbrave150: efficacy and safety results from a ph III study evaluating atezolizumab (atezo) + bevacizumab (bev) vs sorafenib (Sor) as first treatment (tx) for patients (pts) with unresectable hepatocellular carcinoma (HCC). Ann Oncol. 2019;30(Suppl 9):ix186-7. https://doi.org/10.1093/annonc/mdz446.

11.• Finn RS, Qin S, Ikeda M, Galle PR, Ducreux M, Kim T-Y, et al. Atezolizumab plus bevacizumab in unresectable hepatocellular carcinoma. N Engl J Med. 2020;382:1894-905. https://doi.org/10. 1056/NEJMoa1915745 Pivotal recently published data from the IMBrave-150 trial showing higher overall survival compared to sorafenib.

12. Galle PR, Finn RS, Qin S, Ikeda M, Zhu AX, Kim T-Y, et al. Patient-reported outcomes (PROs) from the phase III IMbrave150 trial of atezolizumab (atezo) +bevacizumab (bev) vs sorafenib (sor) as first-line treatment (tx) for patients (pts) with unresectable hepatocellular carcinoma (HCC). J Clin Oncol. 2020;38(4_suppl):476.

13. Llovet JM, Ricci S, Mazzaferro V, Hilgard P, Gane E, Blanc JF, et al. Sorafenib in advanced hepatocellular carcinoma. N Engl J Med. 2008;359:378-90. https://doi.org/10.1056/NEJMoa0708857.

14. Cheng A-L, Kang Y-K, Chen Z, Tsao CJ, Qin S, Kim JS, et al. Efficacy and safety of sorafenib in patients in the Asia-Pacific region with advanced hepatocellular carcinoma: a phase 3 randomised, double-blind, placebo-controlled trial. Lancet Oncol. 2009;10:25-34. https://doi.org/10.1016/S1470-2045(08)70285-7.

15. Kudo M, Finn RS, Qin S, Han KH, Ikeda K, Piscaglia F, et al. Lenvatinib versus sorafenib in first-line treatment of patients with unresectable hepatocellular carcinoma: a randomized phase 3 noninferiority trial. Lancet. 2018;391:1163-73. https://doi.org/10. 1016/S0140-6736(18)30207-1.

16. Vogel A, Frenette C, Sung MW, et al. Baseline liver function and outcomes in the phase III REFLECT study in patients with unresectable hepatocellular carcinoma (uHCC). J Clin Oncol. 2020;38(4_suppl):524.

17. Bruix J, Qin S, Merle P, Granito A, Huang YH, Bodoky G, et al. Regorafenib for patients with hepatocellular carcinoma who progressed on sorafenib treatment (RESORCE): a randomised, double-blind, placebo-controlled, phase 3 trial. Lancet. 2017;389: 56-66. https://doi.org/10.1016/S0140-6736(16)32453-9.

18. Finn RS, Merle P, Granito A, Huang YH, Bodoky G, Pracht M, et al. Outcomes of sequential treatment with sorafenib followed by regorafenib for HCC: additional analyses from the phase III RESORCE trial. J Hepatol. 2018;69:353-8. https://doi.org/10. 1016/j.jhep.2018.04.010.

19. Abou-Alfa GK, Meyer T, Cheng AL, El-Khoueiry AB, Rimassa L, Ryoo BY, et al. Cabozantinib in patients with advanced and progressing hepatocellular carcinoma. N Engl J Med. 2018;379: 54-63. https://doi.org/10.1056/NEJMoa1717002.

20. Zhu AX, Park JO, Ryoo B-Y, Yen CJ, Poon R, Pastorelli D, et al. Ramucirumab versus placebo as second-line treatment in patients with advanced hepatocellular carcinoma following first-line therapy with sorafenib (REACH): a randomised, double-blind, multicentre, phase 3 trial. Lancet Oncol. 2015;16:859-70. https://doi.org/10. 1016/S1470-2045(15)00050-9.

21. Zhu AX, Kang YK, Yen CJ, Finn RS, Galle PR, Llovet JM, et al. Ramucirumab in advanced hepatocellular carcinoma and elevated 
alpha-fetoprotein following sorafenib (REACH-2): a randomised, double-blind, placebo controlled phase 3 trial. Lancet Oncol. 2019;20:282-96. https://doi.org/10.1016/S1470-2045(18)30937-9.

22. U.S. Food and Drug Administration. Center for Drug Evaluation and Research. "FDA grants accelerated approval to nivolumab for HCC previously treated with sorafenib.” U.S. FDA, 25 Sept. 2017, https://www.fda.gov/drugs/resources-information-approved-drugs/ fda-grants-accelerated-approval-nivolumab-hcc-previouslytreated-sorafenib. Accessed 25 April 2020.

23. U.S. Food and Drug Administration. Center for Drug Evaluation and Research. "The US Food and Drug Administration FDA grants accelerated approval to pembrolizumab for hepatocellular carcinoma." U.S. FDA, 14 Dec. 2018, https://www.fda.gov/drugs/fdagrants-accelerated-approval-pembrolizumab-hepatocellularcarcinoma. Accessed 25 April 2020.

24. El-Khoueiry AB, Sangro B, Yau T, Crocenzi TS, Kudo M, Hsu C, et al. Nivolumab in patients with advanced hepatocellular carcinoma (CheckMate 040): an open-label, non-comparative, phase 1/2 dose escalation and expansion trial. Lancet. 2017;389:2492-502. https://doi.org/10.1016/S0140-6736(17)31046-2.

25. Bristol-Myers Squibb. Bristol-Myers Squibb announces results from CheckMate-459 study evaluating Opdivo (nivolumab) as a first-line treatment for patients with unresectable hepatocellular carcinoma. Princeton, NJ: Bristol Myers Squibb; 2019 [updated June 24, 2019]. Available online: https://news.bms.com/pressrelease/bmy/bristolmyers-squibb-announces-results-checkmate459-studyevaluating-opdivo-nivol. Accessed 25 April 2020.

26. Edeline J, Yau T, Park J-W, Kudo M, Han K-H, Mathurin R, et al. CheckMate 459: health-related quality of life (HRQoL) in a randomized, multicenter phase III study of nivolumab (NIVO) versus sorafenib (SOR) as first-line (1L) treatment in patients (pts) with advanced hepatocellular carcinoma (aHCC). J Clin Oncol. 2020;38(4_suppl):483.

27. Zhu AX, Finn RS, Edeline J, Cattan S, Ogasawara S, Palmer D, et al. Pembrolizumab in patients with advanced hepatocellular carcinoma previously treated with sorafenib (KEYNOTE-224):a nonrandomised, open-label phase 2 trial. Lancet Oncol. 2018;19: 940-52. https://doi.org/10.1016/S1470-2045(18)30351-6.

28. Kudo M, Finn RS, Edeline J, et al. Updated efficacy and safety of KEYNOTE-224: a phase II study of pembrolizumab (pembro) in patients with advanced hepatocellular carcinoma (HCC). J Clin Oncol. 2020;38(4_suppl):518.

29. Finn RS, Ryoo BY , Merle P, Kudo M, Bouattour M, Lim HY, et al. KEYNOTE-240 investigators. Pembrolizumab as second-line therapy in patients with advanced hepatocellular carcinoma in KEYNOTE-240: a randomized, double-blind, phase III trial. J Clin Oncol. 2020;38(3):193-202. https://doi.org/10.1200/JCO.19. 01307.

30. Kudo M, Lim HY, Cheng AL, et al. Phase III study of pembrolizumab (pembro) versus best supportive care (BSC) for second-line therapy in advanced hepatocellular carcinoma (aHCC): KEYNOTE-240 Asian subgroup. J Clin Oncol. 2020;38(4_suppl):526.

31. U.S. Food and Drug Administration. Center for Drug Evaluation and Research. "FDA grants accelerated approval to nivolumab and ipilimumab combination for hepatocellular carcinoma." U.S. FDA, 11 Mar. 2020, https://www.fda.gov/drugs/resources-informationapproved-drugs/fda-grants-accelerated-approval-nivolumab-andipilimumab-combination-hepatocellular-carcinoma. Accessed 8 June 2020.

32. Yau T, Kang YK, Kim TY, et al. Nivolumab + ipilimumab combination therapy in patients with advanced hepatocellular carcinoma: results from CheckMate 040. J Clin Oncol. 2019;37(15_suppl): 4012.

33. He AR, Yau T, Hsu C, et al. Nivolumab (NIVO) + ipilimumab (IPI) combination therapy in patients (pts) with advanced hepatocellular carcinoma (aHCC): subgroup analyses from CheckMate 040. J Clin Oncol. 2020;38(4_suppl):512.

34. Yau T, Zagonel V, Santoro A, et al. Nivolumab (NIVO) + ipilimumab (IPI) + cabozantinib (CABO) combination therapy in patients (pts) with advanced hepatocellular carcinoma (aHCC): results from CheckMate 040. J Clin Oncol. 2020;38(4_suppl):478.

35. Kudo M, Ikeda M, Motomura K, et al. A phase Ib study of lenvatinib (LEN) plus nivolumab (NIV) in patients (pts) with unresectable hepatocellular carcinoma (uHCC): study 117 . J Clin Oncol. 2020;38(4 suppl):513 Important ongoing trial investigating the combination of tyrosine kinase inhibitor, lenvatinib, in combination with anti-PD-1, nivolumab.

36. Gosain R, Mukherjee S, Lee SS, et al. Phase Ib/II study of sorafenib (SOR) and pembrolizumab (PEM) in advanced hepatocellular cancer (HCC). J Clin Oncol. 2020;38(4_suppl):TPS596 Important ongoing trial investigating the combination of tyrosine kinase inhibitor, sorafenib, in combination with anti-PD-1, pembrolizumab.

37. Welling T, Beri N, Siolas D, et al. A phase II, randomized, controlled trial of nivolumab in combination with BMS-986253 or cabiralizumab in advanced hepatocellular carcinoma (HCC) patients. J Clin Oncol. 2020;38(4_suppl):TPS598.

38. Ogasawara S, Kanzaki H, Koroki K, et al. Phase I study of a new concept cancer vaccine composed artificial intelligence (AI)-designed shared-antigen peptides plus combined synergistically activating antigen-specific CTL reaction (CYT001) in patients with advanced hepatocellular carcinoma (CRESCENT 1). J Clin Oncol. 2020;38(4_suppl):TPS595.

39. National Institute of Public Health of Japan. A Phase I Study of CYT001. NIPH Clinical Trials Search. https://rctportal.niph.go.jp/ en/detail?trial id=jRCT2031190072. Accessed 25 March 2020.

40. Birge R, Boeltz S, Kumar S, et al. Phosphatidylserine is a global immunosuppressive signal in efferocytosis, infectious disease, and cancer. Cell Death Differ. 2016;23:962-78. https://doi.org/10. 1038/cdd.2016.11.

41. Beg MS, Zhu H, Hseih D, et al. A phase II clinical trial of the phosphatidylserine targeting antibody, bavituximab, in combination with pembrolizumab in patients with advanced hepatocellular carcinoma. J Clin Oncol. 2020;38(4 suppl):TPS599.

42. Harding JJ, Abou-Alfa GK, Shi Y, et al. A phase II randomized placebo-controlled study investigating the combination of yiv-906 and sorafenib (SORA) in HBV (+) patients (Pts) with advanced hepatocellular carcinoma (HCC). J Clin Oncol. 2020;38(4_suppl): TPS601.

43. Chu E. Wedding rigorous scientific methodology and ancient herbal wisdom to benefit cancer patients: the development of PHY906. Oncology (Williston Park). 2018;32(2):e20-7.

44. Breitbach CJ, Moon A, Burke J, Hwang T-H, Kirn DH. A phase 2, open-label, randomized study of Pexa-Vec (JX-594) administered by intratumoral injection in patients with unresectable primary hepatocellular carcinoma. Methods Mol Biol. 2015;1317:34357. https://doi.org/10.1007/978-1-4939-2727-2_19 Novel ongoing trial investigating oncolytic and immunotherapeutic vaccina virus therapy combined with sorafenib.

45. Qin S, Finn RS, Kudo M, Meyer T, Vogel A, Ducreux M, et al. RATIONALE 301 study: tislelizumab versus sorafenib as first-line treatment for unresectable hepatocellular carcinoma. Future Oncol. 2019;15(16):1811-22. https://doi.org/10.2217/fon-2019-0097.

46. Deva S, Lee J-S, Lin C-C, et al. A phase IA/IB trial of tislelizumab, an anti-PD-1 antibody, in patients with advanced solid tumors. Ann Oncol. 2018;29(suppl_8):x24-5.

47. Abou-Alfa GK, Chan SL, Furuse J, Galle PR, et al. A randomized, multicenter phase 3 study of durvalumab (D) and tremelimumab (T) as first-line treatment in patients with unresectable hepatocellular carcinoma (HCC): HIMALAYA. J Clin Oncol. 2018;36(15_suppl):TPS4144 Important ongoing trial 
investigating the combination of PD-L1 inhibitor, durvalumab, in combination with anti-CTLA-4, tremelimumab.

48. Viña $G$ "Imfinzi and tremelimumab granted Orphan Drug Designation in the US for liver cancer." AstraZeneca; 20 Jan 2020. https://www.astrazeneca.com/media-centre/pressreleases/2020/imfinzi-and-tremelimumab-granted-orphan-drugdesignation-in-the-us-for-liver-cancer-20012020.html. Accessed 8 June 2020

49. Kelley RK, Cheng AL, Braiteh FS, Park JW, et al. Phase 3 (COSMIC-312) study of cabozantinib (C) in combination with atezolizumab (A) versus sorafenib (S) in patients (pts) with advanced hepatocellular carcinoma (aHCC) who have not received previous systemic anticancer therapy. J Clin Oncol. 2019;37(15_suppl):TPS4157 Important ongoing trial investigating the combination of tyrosine kinase inhibitor, cabozantinib, in combination with anti-PD-L1, atezolizumab.

50. Llovet JM, Kudo M, Cheng AL, et al. Lenvatinib (len) plus pembrolizumab (pembro) for the first-line treatment of patients (pts) with advanced hepatocellular carcinoma (HCC): phase 3 LEAP-002 study. J Clin Oncol. 2019;37(15_suppl):TPS4152 Important ongoing trial investigating the combination of tyrosine kinase inhibitor, lenvatinib, in combination with anti-PD1, pembrolizumab.

51. Llovet JM, Finn RS, Ikeda M, et al. A phase $1 \mathrm{~b}$ trial of lenvatinib (LEN) plus pembrolizumab (PEMBRO) in unresectable hepatocellular carcinoma (uHCC): updated results. Ann Oncol. 2019;30(suppl_5):v253-324. https://doi.org/10.1093/annonc/ mdz247.
52. El-Khoueiry A, Spano JP, Angevin E, et al. Analysis of OX40 agonist antibody (PF-04518600) in patients with hepatocellular carcinoma. J Clin Oncol. 2020;38(4_suppl):523.

53. Sarker D, Plummer ER, Basu B, Meyer T, Huang K-W, Evans TRJ. Preliminary results of a first-in-human, first-in-class phase I study of MTL-CEBPA, a small activating RNA (saRNA) targeting the transcription factor $\mathrm{C} / \mathrm{EBP}-\alpha$ in patients with advanced liver cancer. J Clin Oncol. 2018;36(15_suppl):2509.

54. Sarker D, Sodergren M, Plummer ER, et al. First-in-human phase I trial of small activating RNA (saRNA) oligonucleotide MTLCEBPA in combination with sorafenib in patients with advanced hepatocellular carcinoma (HCC). J Clin Oncol. 2020;38(4_suppl): 554.

55. Skill NJ, Maluccio MA. Apurinic/apyrimidinic endonuclease-1 and hepatocellular carcinoma. J Clin Oncol. 2020;38(4_suppl):563.

56. Wakizaka K, Kamiyama T, Orimo T, Shimada S, Nagatsu A, Asahi $\mathrm{Y}$, et al. Role of Wnt5a in suppressing invasiveness of hepatocellular carcinoma via epithelial mesenchymal transition. J Clin Oncol. 2020;38(4_suppl):573.

57. Monga S, Borad MJ, Tao J. Beta-catenin mutations in hepatocellular cancer, tumor cell metabolism, and the response of these tumors to mTOR inhibition. J Clin Oncol. 2020;38(4_suppl):583.

Publisher's Note Springer Nature remains neutral with regard to jurisdictional claims in published maps and institutional affiliations. 